\section{Synthesis of Maximally Sparse Arrays Using Compressive Sensing and Full-Wave Analysis for Global Earth Coverage Applications}

\author{
C. Bencivenni, M. V. Ivashina, Senior Member, IEEE, \\ R. Maaskant, Senior Member, IEEE, and J. Wettergren
}

\begin{abstract}
Global optimization methods can be employed to design aperiodic array antennas that accurately account for their electromagnetic behavior and complex performance specifications. However, they are computationally expensive and therefore limited to small to mid-sized array problems. On the other hand, analytical methods do not suffer from this problem, but often assume idealized antenna elements and fully adjustable excitation controls, thereby excluding beam degradation effects caused e.g. by mutual coupling and quantized phase shifters. We present a fast design method for large maximally sparse arrays (MSAs) that is capable of handling the aforementioned limitations. It is based on the previously published combined EM-Compressive Sensing approach which has been herein generalized for multibeam optimization, and where we also exploit array symmetry in order to reduce the design complexity. Results are obtained for a circular array ( $100 \lambda$ diameter) of horn antennas operating in a multi-beam SATCOM scenario, and demonstrate that even weak mutual coupling effects and small phase quantization are important when very demanding side-lobe and cross-polarization levels are required.
\end{abstract}

Index Terms-sparse array antennas, multibeam antennas, satellite applications.

\section{INTRODUCTION}

Aperiodic array antennas are of great interest for modern array applications, thanks to their ability to enhance multi-beam performance while minimizing the number of elements and associated costs with respect to classical uniform arrays [1]. To realize such array designs, Global Optimization (GO) and, generally, stochastic techniques have been widely used [2]-[5]. Although well-established, these methods are computationally expensive, and thereby suitable only for small to medium sized array problems, or be used as a refinement of an initial solution for large arrays [6]. Analytical techniques, on the other hand, are very effective in handling large problems [7][13]. A common drawback of the latter methods is that they assume idealized antenna elements (often isotropic radiators) and, hence, exclude mutual coupling (MC) effects between antenna ports, which may be inadequate in some cases. The resulting degradation of the beam quality (such as increased side-lobe [14] and cross-polarization levels) can be assessed by means of an a posteriori full-wave simulation of the final array [15]. Accordingly, the effective steering vector can be found by multiplying the antenna mutual coupling matrix with the initially obtained steering vector [16]. Unfortunately, the

C. Bencivenni, M. V. Ivashina, and R. Maaskant are with the Signals and Systems Department of the Chalmers University of Technology, Göteborg, Sweden, e-mail: carlo.bencivenni@chalmers.se, marianna.ivashina@chalmers.se, rob.maaskant@chalmers.se. J. Wettergren is with RUAG Aerospace Sweden, Göteborg, Sweden, johan.wettergren@ruag.com.

This work is financed by Swedish VR and VINNOVA grants.

Manuscript received April xx, 20xx; revised January xx, 20xx.

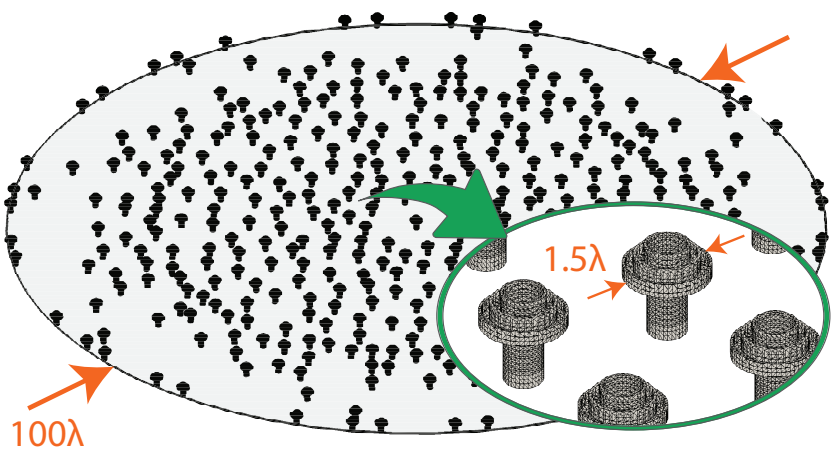

Fig. 1: Full-wave EM model (CBFM) of the synthesized array comprising 385 pipe horn antenna elements.

latter approach is limited to minimum scattering antennas, and is neither part of the optimization loop.

Another important aspect that is often neglected, particularly in analytical methods, is the joint optimization for multiple beams. In fact, the array layout is often optimized for a single (broadside) beam, which may not be a severe restriction for small beam-scanning scenarios [17]. For wider scan ranges, the beam scanning degradation due to a non-uniform element pattern can be accounted for by modifying the optimization mask [18].

The goal of this work is to go further and to develop a fast design method for large maximally sparse arrays (MSAs) - i.e. arrays with the smallest number of antenna elements satisfying a given pattern mask for multiple scanned beams while accurately accounting for electromagnetic coupling effects and phase shifter quantization in the optimization phase. Our approach is based on the Compressive Sensing theory, where the synthesis of MSAs is formulated as the problem of finding the element excitation vector with a minimum number of non-zero entries (while fulfilling certain pattern constraints). This is accomplished through an iterative convex optimization procedure [19]. We have: (i) extended the original formulation, which is constrained to single-beam arrays of isotropic elements radiating scalar fields, to the multi-beam vectorial-field scenario; and (ii) hybridized the original iterative optimization procedure with a full-wave EM analysis to include the effects of the antenna element geometry and mutual coupling into the design process (cf. Fig. 1). This hybridization represents also an extension to [14] from linear to planar arrays, where we can enforce a certain degree of symmetry of the array layout to reduce the design complexity, which can subsequently be exploited to reduce the optimization time.

This paper is organized as follows. Section II presents the extended formulation of the MSA optimization problem and describes the iterative EM-analysis and combined convex optimization procedure. In Sec. III, the developed approach is examined for large planar arrays comprising horn antenna elements and operating in a multi-beam scenario. The performance of the algorithm is assessed in terms of the beam characteristics, number of array elements, as well as overall synthesis time. Array symmetry, beam scanning and mutual coupling effects are discussed in separate subsections. The conclusions are presented in Sec. IV. 


\section{Methodology}

\section{A. Generic Formulation of the Optimization Problem}

The problem of synthesising a maximally sparse array is that of designing an array with the least number of elements. This can also be stated as finding the excitation vector $\mathbf{w}=\left[w_{1}, w_{2}, \ldots, w_{N}\right]^{T}$ (where ${ }^{T}$ denotes the transpose) with the minimum number of non-zero entries, that is, to solve the problem argmin $\|\mathbf{w}\|_{\ell_{0}}$ with $\mathbf{w} \in \mathbb{C}^{N}$, subject to certain pattern constraints. This is a NP-hard (Nondeterministic Polynomial time hard [20]) problem for which no closed-form solution exists, and finding a solution using a combinatorial search method is intractable, even for moderate array sizes. It is, however, possible to obtain a solution to this problem through a more tractable iterative convex optimization procedure. In [19], the problem is relaxed and solved in a semi-analytical manner by approximating the $\ell_{0}$-norm minimization through an iterative weighted $\ell_{1}$-norm minimization procedure.

As a generalization to [19] and [14], for $P$ focused beam patterns scanning at the directions $\left\{\hat{\boldsymbol{r}}_{p}\right\}_{p=1}^{P}$ and prescribed radiation masks $\left\{M_{\nu}^{p}(\hat{\boldsymbol{r}})\right\}_{p=1}^{P}$ for the vector field component $\nu$, the $i^{\text {th }}$ iteration of the algorithm reads

$$
\begin{aligned}
& \underset{\mathbf{w}^{i} \in \mathbb{C}^{N}}{\operatorname{argmin}}\left\|\mathbf{Z}^{i} \mathbf{w}^{i}\right\|_{\ell_{1}} \\
& \text { subject to } \begin{cases}f_{\mathrm{co}}^{p}\left(\hat{\boldsymbol{r}}_{\mathrm{p}}\right)=1, & p=1 \\
\left|f_{\nu}^{p}(\hat{\boldsymbol{r}})\right|^{2} \leq M_{\nu}^{p}(\hat{\boldsymbol{r}}), & p=1, \ldots, P\end{cases}
\end{aligned}
$$

where, in the minimization objective, $\mathbf{Z}^{i}$ is a diagonal matrix with entries $\mathbf{z}^{i}=1 /\left(\left|\mathbf{w}^{(i-1)}\right|+\epsilon\right)$, chosen as to maximally enhance the sparsity of the solution and is selected based on the recommendation of [14]. The constraint terms involve the array far-field patterns, which for $N$ elements with locations $\left\{\boldsymbol{r}_{n}\right\}_{n=1}^{N}$, excitation coefficients $\left\{w_{n}\right\}_{n=1}^{N}$ and embedded far-field vector patterns $\left\{\boldsymbol{f}_{n}(\hat{\boldsymbol{r}})\right\}_{n=1}^{N}$ can be generally written as $\boldsymbol{f}(\hat{\boldsymbol{r}})=\sum_{n=1}^{N} w_{n} \boldsymbol{f}_{n}(\hat{\boldsymbol{r}})=f_{\mathrm{co}}(\hat{\boldsymbol{r}}) \hat{\mathbf{c o}}+$ $f_{\mathrm{xp}}(\hat{\boldsymbol{r}}) \hat{\mathbf{x p}}=\left[\mathbf{w}^{T} \mathbf{f}_{\mathrm{co}}(\hat{\boldsymbol{r}})\right] \hat{\mathbf{c o}}+\left[\mathbf{w}^{T} \mathbf{f}_{\mathrm{xp}}(\hat{\boldsymbol{r}})\right] \hat{\mathbf{x p}}$, where $\mathbf{f}_{\nu}=$ $\left[f_{\nu, 1}, f_{\nu, 2}, \ldots f_{\nu, N}\right]^{T}$ for $\nu \in\{\mathrm{co}, \mathrm{xp}\}$ are the co-polar and cross-polar steering vectors.

Additionally, in multi-beam phased arrays, beams are scanned by applying an appropriate phase shift to each element excitation while keeping its magnitude fixed. Hence, for a generic beam $p$ with far-field function $\boldsymbol{f}^{p}(\hat{\boldsymbol{r}})$, the corresponding excitation coefficients can be expressed as $w_{n}^{p}=w_{n} e^{-j \Phi_{n}^{p}}$, where $\Phi_{n}^{p}$ is the phase shift at element $n$ for beam $p$. For linear phase shift scanning in the direction $\hat{\boldsymbol{r}}_{p}$, the corresponding phase shift term is $\Phi_{n}^{p}=k \boldsymbol{r}_{n} \cdot \hat{\boldsymbol{r}}_{p}$. However, quantized phase shifters and other non-idealities can affect such values. To account for this, each phase shift term can be modified accordingly, e.g. by appropriate rounding leading to quantized phase shifters, and be included in the design phase.

Please note that the method returns both the element position and excitations, thus the final array is aperiodic but not isophoric.

\section{B. Iterative Optimization Procedure}

To include the effects of mutual coupling (MC), a two step iterative optimization procedure is used [14], cf. Fig. 2. During

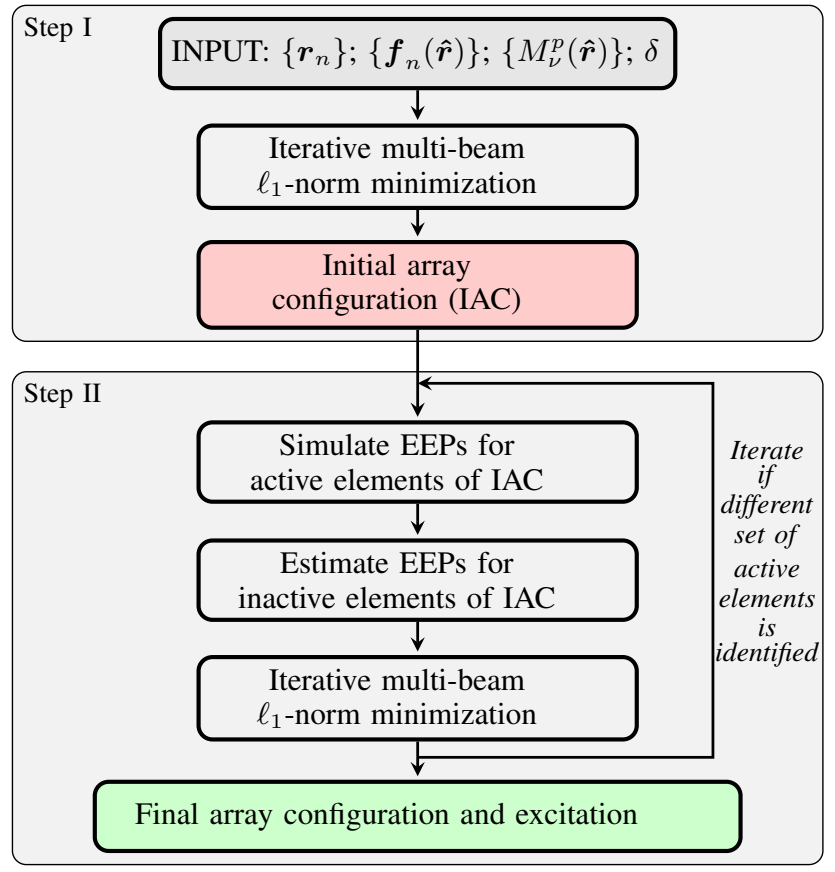

Fig. 2: Block diagram of the proposed optimization approach.

Step I, the optimization is performed without considering the $\mathrm{MC}$ effects and yields an initial sparse array configuration while Step II involves the iterative full-wave EM analysis to refine this initial solution. The available aperture is discretized very finely so as to emulate a quasi-continuous element positioning and phase-shifted versions of the simulated isolated element pattern (IEP) are assumed. Once the algorithm converges, the significant (active) elements are found by setting a threshold $\delta$ on the excitation magnitudes.

In Step II, a full-wave analysis of the initial array is performed to obtain embedded element patterns (EEPs) of the active elements. The IEPs of the active elements are then replaced with the simulated EEPs, while the patterns of the remaining elements are estimated by assuming a phaseshifted version of their nearest simulated EEP. With this new set of element patterns, the algorithm is invoked again to obtain a new array layout. This procedure is repeated until the convergence criterion is satisfied, i.e, the state of active and inactive elements remains the same between two consecutive iterations. Typically, few MoM- $\ell_{1}$ iterations are needed to reach convergence.

\section{RESULTS}

In the following subsection we describe how the symmetry of the array layout can be enforced and subsequently be exploited to reduce the optimization time and design complexity. We then present numerical results obtained with the developed multi-beam optimization procedure for SATCOM applications whose specifications are summarized in Table I and where the beam coverage regions are as shown in Fig. 3. For this type of application, frequency reuse is very important, giving very stringent requirements on both the side-lobe and crosspolarization levels of each beam in the Field of View (FoV). 


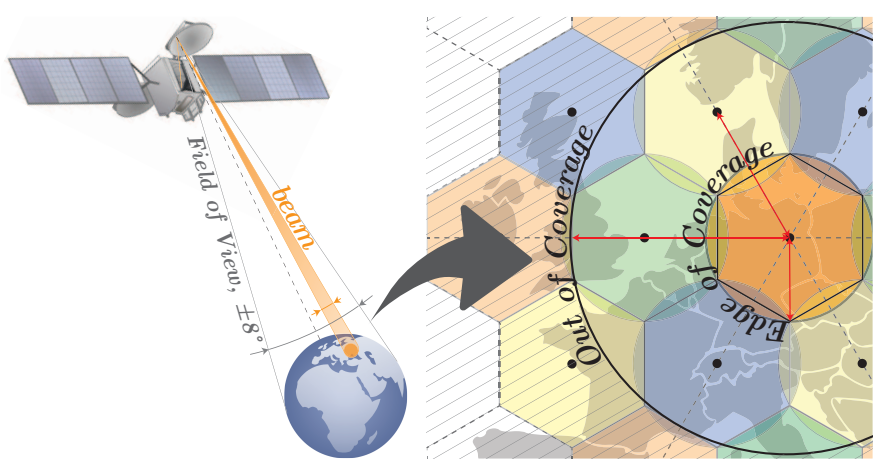

Fig. 3: Illustration of the multi-beam scenario, where (see insertion on the right) each hexagon represents a (main-lobe) beam spot on the Earth and the colors distinguish the four frequency bands.

Typical values are $25-27 \mathrm{~dB}$ below the desired signal level. However, relaxed requirements exist for the Out of the Earth coverage, since spurious radiation in this region does not lead to signal interference between beams but solely to a loss in gain, and an increased system noise temperature in receive mode, which is negligible owing to the cold space. Hence, the aim is to minimize the number of array elements, while fulfilling the side-lobe and cross-polarization requirements. Accordingly, the pattern mask used in our optimization for the maximum side-lobe and cross-polarization levels is set to $-25 \mathrm{~dB}$ from the Out of Coverage $\left( \pm 0.795^{\circ}\right)$ up to the edge of the Field-Of-View region $\left( \pm 8^{\circ}\right)$.

The considered array element in Fig. 4 is a circular corrugated pipe horn designed by RUAG Space AB [21]. The element design frequency is $18.3 \mathrm{GHz}$, at which its aperture diameter equals $1.5 \lambda$. Note that, over the given $\mathrm{FoV}$, this element has a virtually constant directivity of about $9 \mathrm{dBi}$ and D-plane relative cross-polarization level of order $-40 \mathrm{~dB}$.

\section{A. Exploitation of the Array Symmetry}

By enforcing symmetries in the array layout the overall system design renders to a modular design approach, which is beneficial for reducing the manufacturing costs as well as the complexity of the connected subsystems. In fact, since symmetry is enforced also on the excitation coefficients, only a reduced number of amplifier types is needed. Furthermore, symmetry can be exploited to expedient the optimization process. In this subsection we consider the single beam optimization case. Although various kinds of symmetries may be enforced, we focus on rotational symmetry, since circularly symmetric beams are required for the given SATCOM

TABLE I: Specification for the antenna design optimization

\begin{tabular}{ll}
\hline Array type & Planar, dual-polarized \\
Antenna element type & Corrugated pipe horn \\
Field of View (FoV) & $\pm 8^{\circ}$ \\
Beam arrangement & Multi-spot 4-band hexagonal grid \\
Interbeam distance & $1.06^{\circ}$ \\
Edge of Coverage angle & $0.61^{\circ}$ \\
Out of Covererage (OoC) angle & $0.795^{\circ}$ \\
Max. SLL in the OoC region & $-25 \mathrm{~dB}$ \\
\hline
\end{tabular}

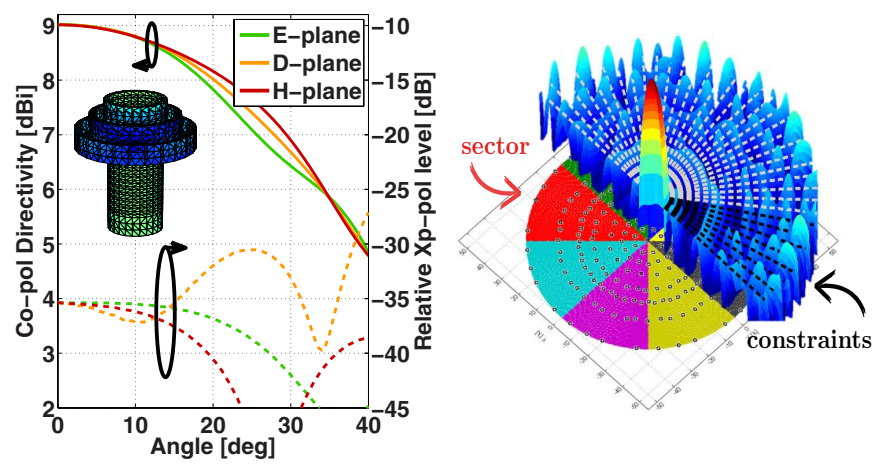

Fig. 4: The corrugated horn (left, inset): MoM model and its isolated element pattern; (right) The array layout and pattern for the case of the 8-fold symmetry, where only one aperture and pattern sector are considered during the optimization.

application. Rotational symmetry of order $N_{\text {sym }}$ is obtained by subdividing the circular aperture of the array in $N_{\text {sym }}$ sectors. This subdivision allows one to consider only the base sector of the array in step I of the optimization process (no MC yet), while the remaining part of the layout can be obtained through rotation operations on the sectors and element patterns. This reduces the number of discretization points of the uniform array, and hence, the number of optimization unknowns. Furthermore, since the resulting pattern will also exhibit such symmetry, the number of pattern samples, and therefore the number of constraints to accurately describe the pattern mask $M_{\nu}^{p}(\hat{\boldsymbol{r}})$, can be reduced as well. Figure 4 depicts the symmetry in the array layout as well as in the resulting pattern in case of an 8-fold symmetry, as an example.

Table II shows the results for the 4-, 8-, 16- and 32-fold rotational symmetries. For each case, the resulting array layout, including its corresponding base sector, and 3D-pattern for the broadside-scanned beam are shown. As can be seen, the design time is very much reduced by increasing the order of symmetry, albeit at the cost of the increased number of array elements (and thus distinct element weights). For the application at hand, the 8-fold symmetry provided the best choice offering a significantly reduced design time (factor 64) with only a moderate increase in the element number (4\%) as compared to the reference layout (w/o exploiting symmetry).

Additionally, the robustness of the method has been studied in terms of the aperture sampling density. Results indicate that a sampling distance of about $0.08 \lambda-0.03 \lambda$ is an adequate choice for achieving an optimal freedom in element placement, design robustness and computational complexity.

\section{B. Optimization for Multiple Beams}

Next, we discuss the achieved beam-scanning performance when simultaneously optimizing the array layout for multiple beams, and compare the results to those for the single beam optimized case. As in the previous analysis, an 8-fold symmetry is adopted. In conventional phased-array approaches, scanning is a simple phase translation in the array factor and certain beam degradation effects caused by the EEPs and electronic controls are therefore neglected. Let us refer to the 
TABLE II: Comparison of optimal array layouts, obtained by imposing different symmetry orders with respect to the reference case when the entire array aperture is considered in the $\ell_{1}$-norm minimization.

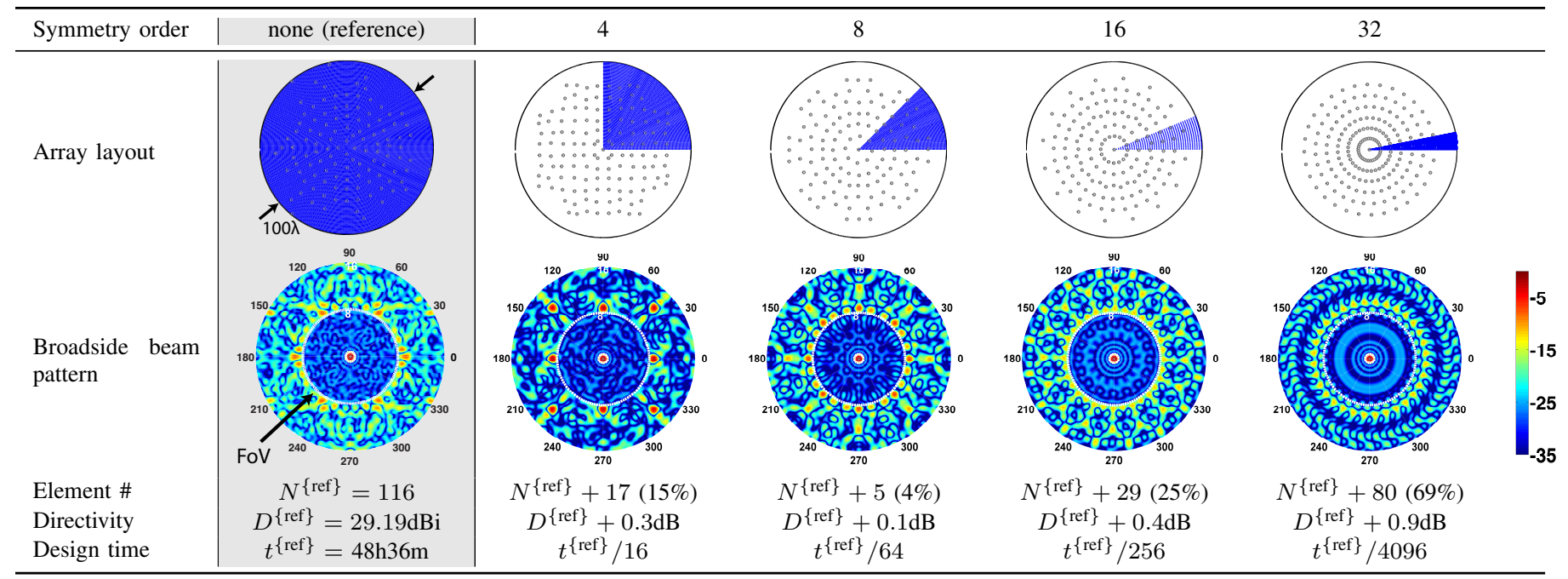

Reference approach as the single beam broadside optimized case. The SLL mask covers a suppression region twice larger then the FoV $\left( \pm 16^{\circ}\right)$ to guarantee the desired SLL when scanning within $\pm 8^{\circ}$. It is worth pointing out that the extended radiation mask can be corrected such as to include the effect of the element pattern shape [18], however, for the present element the IEP is almost uniform over the FoV, its effect will be limited. The proposed multi-beam approach intrinsically designs arrays by simultaneously optimizing for multiple beam directions and masks. To demonstrate this, two arrays have been designed, with and without accounting for a $10^{\circ}$ phase shifter quantization.

Figures 5(a)-(c) present the comparison results for the worst-case scanning situation, i.e, when the scan angle corresponds to the edge of the field-of-view (FoV) in the diagonal plane. The optimized array for broadside operation is unsuitable for scanning to such large angles, as the $18.2 \mathrm{~dB}$ peak side-lobe level in Fig. 5(a) largely exceeds the required $-25 \mathrm{~dB}$ level within the FoV. The far-field pattern of the synthesized array in Fig. 5(b) satisfies the mask constraints with a maximum relative SLL increase of $2.3 \mathrm{~dB}$ when phase shifter quantization is not accounted for, and less than $0.7 \mathrm{~dB}$ otherwise [cf. Fig. 5(c)]. This performance improvement is owing to the fact that the pattern mask constraints for both the broadside and the outermost scanned beams were imposed, albeit at the cost of an increased number of elements.

We have found that this two-beam optimization procedure is adequate choice to achieve the desired accuracy and keep the optimization time to minimum (due to the increased number of constraints) relatively to the single beam optimization case. Notice that these arrays have different element excitation amplitudes (are not isophoric), thus the aperture efficiency is always reduced. The chosen array has a directivity of about $34 \mathrm{dBi}$, only $1 \mathrm{~dB}$ less than the uniformly-excited case. In the latter case, however, the SLL would increase to $-20 \mathrm{~dB}$.

\section{Inclusion of Mutual Coupling Effects}

Mutual coupling effects can play an important role in designing sparse antenna arrays, even in case of weakly coupled elements, such as the considered pipe horn. The following example demonstrates some of these effects for the 8-fold symmetric array optimized for multi-beam applications without phase shifter quantization presented in the previous section, Figure 5(b). It is pointed out that, also for symmetric arrays, the MC modeling introduced in Sec. II-B requires a full-wave analysis of the entire array. The resulting EEPs are then summed to the corresponding base sector EEPs (with a phase term correction when scanning) which yields a set of active base sector EEPs. Note that this is similar to imposing periodic boundary conditions in infinite phased array approaches yielding an active unit cell. This, in turn, enables us to apply the sparsification algorithm to the active base sector only. Although not employed in this manuscript, upon imposing appropriate EM-field boundary conditions one could also exploit the rotational symmetry in reducing the computational burden of the full-wave analysis. The fullwave analysis (CBFM) of the entire array with 385 elements required approximately 20 hours.

The meshed array geometry is shown in Fig. 1 and the resulting EEPs and the IEP of the pipe horn are shown in Fig. 6. The initial element positions and excitations, as well as their relative change after accounting for $\mathrm{MC}$ effects, are listed in Table III: the minimum inter-element distance varies between $2 \lambda$ to $6.4 \lambda$, therefore both densely as well as sparsely separated elements are present in the array. As a result, the EEPs are different and exhibit strong oscillations around the IEP with a ripple of about $\pm 2 \mathrm{~dB}$ for the co- and $\pm 20 \mathrm{~dB}$ for the cross-polarization components, respectively.

The total array patterns, which have been computed from the initially assumed IEPs and the MoM-simulated EEPs are cross-compared in Fig. 7. The co-polarization component (left) suffers from an increase in SLL of about $1 \mathrm{~dB}$, both in the close proximity of the main beam as well as for far-off scanned 


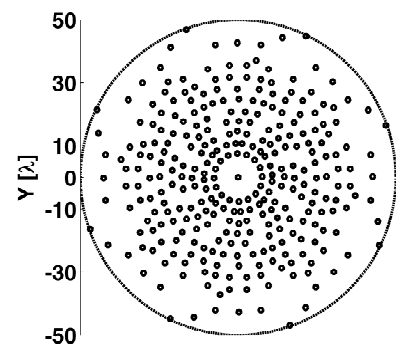

Ele\#: $\mathbf{3 0 5}$

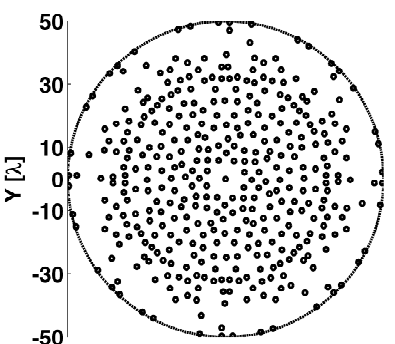

Ele\#: $\mathbf{3 8 5}$

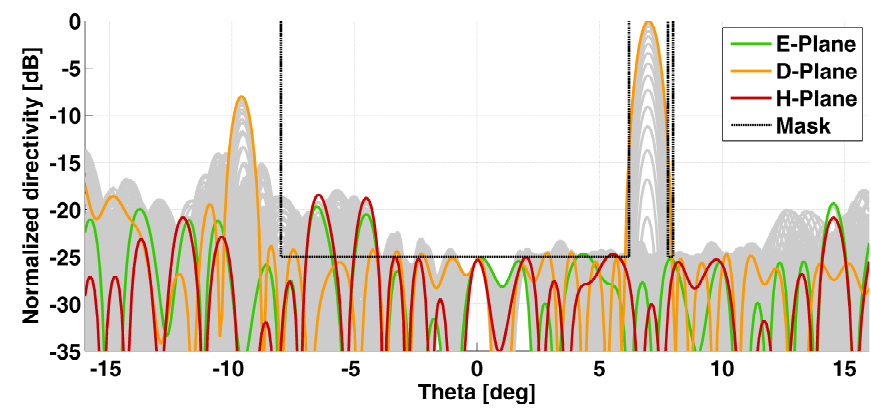

(a) Reference approach

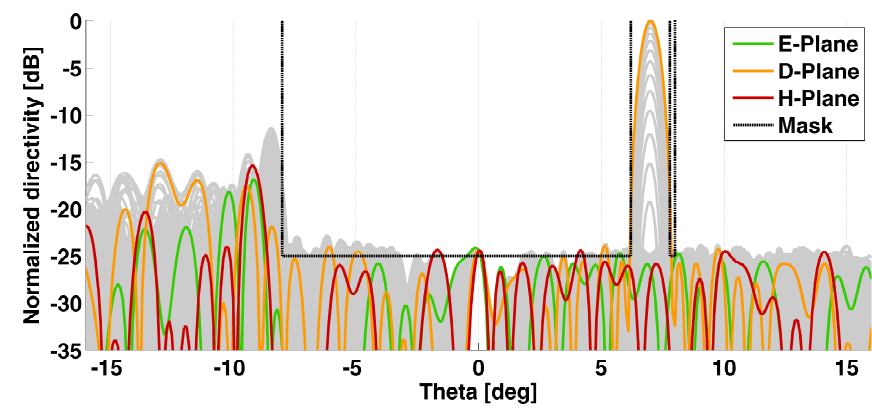

(b) Proposed multi-beam approach: not accounting for phase shifter quantization

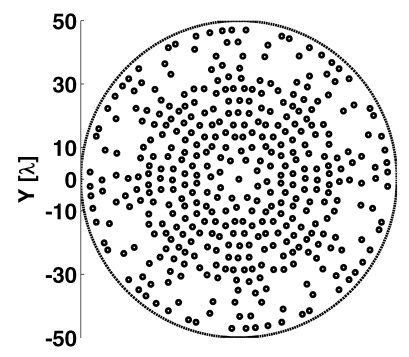

Ele\#: 385

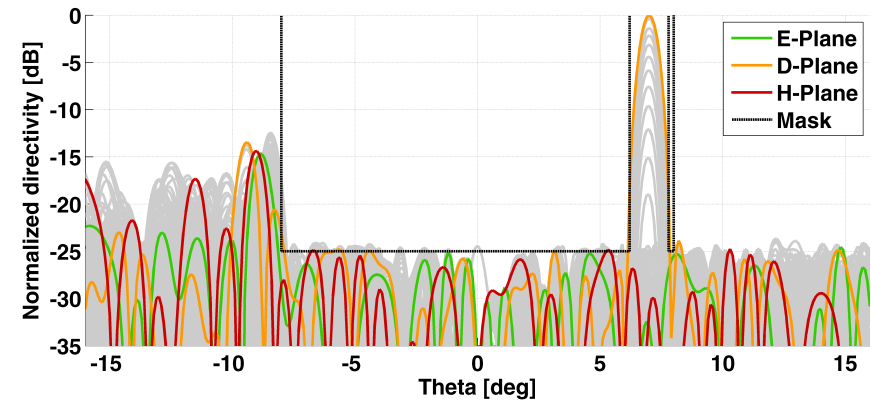

Theta [deg]

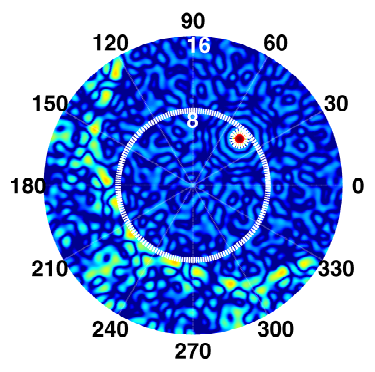

Gain: 34.0dBi

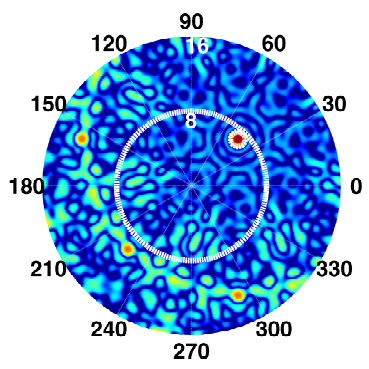

Gain: 32.9dBi

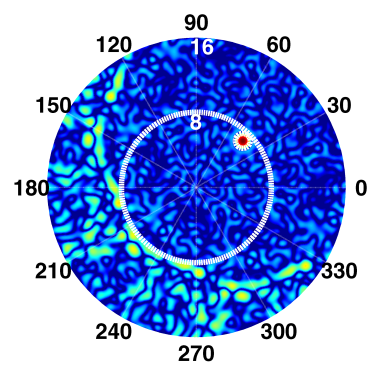

Gain: 34.0dBi

(c) Proposed multi-beam approach: accounting for phase shifter quantization

Fig. 5: Comparison of three array layouts and corresponding scanned beams at $8^{\circ}$ resulting from different optimization strategies: (a) optimization assuming a broadside (BS) beam scenario, where the element excitation vector for the scanned beam has been obtained by multiplying the BS-beam optimized excitation vector with the phase steering factor for $8^{\circ}$, and; (b) the proposed multi-beam approach when not accounting for the phase shifter quantization and (c) when including it in the optimization.

beams. The cross-polarization pattern (right) is affected by an increase of about $10 \mathrm{~dB}$ in the broadside direction and around $30 \mathrm{~dB}$ over the rest of the FoV. It is worth noticing that, despite the strong distortion of the embedded element patterns, the effects on the total co-polar pattern are limited. The algorithm corrects for this distortion with just two additional iterations, while reducing the number of elements from 385 to 361 at the same time. The relative corrections to the base sector are listed as superscripts in Table III. As one can see, two new elements are introduced, 5 elements are removed, 4 elements have their positions adjusted, and all excitations are modified.

On the other hand, the cross-polarization pattern exhibits much higher power levels than for the case when MC effects are ignored, but these levels are still acceptable for the chosen scenario. For applications that are more susceptible to crosspolarization variations, or high cross-polarization levels, it is recommended to include the cross-polar mask constraint levels in the optimization process.

\section{Conclusions}

An array synthesis method has been proposed and demonstrated for the design of circularly symmetric planar arrays, in particular for providing multi-beam global Earth coverage.

It has been shown that: (i) to meet the SLL constraints over the specified 2D-coverage region, the array layout optimization only needs to consider the broadside and an outermost scanned beam; (ii) phase shifter quantization should be accounted for in order to meet the stringent SLL specifications, and that; (iii) MC effects have a strong effect when demanding XP levels are required.

Furthermore, enforcing and exploiting symmetries in the array layout not only allows for a modular design but also reduces the optimization time: a factor 64 has been reached for an 8 -fold rotationally symmetric array case.

\section{REFERENCES}

[1] H. Unz, "Linear arrays with arbitrarily distributed elements," Antennas and Propagation, IRE Transactions on, vol. 8, no. 2, pp. 222-223, 1960. 


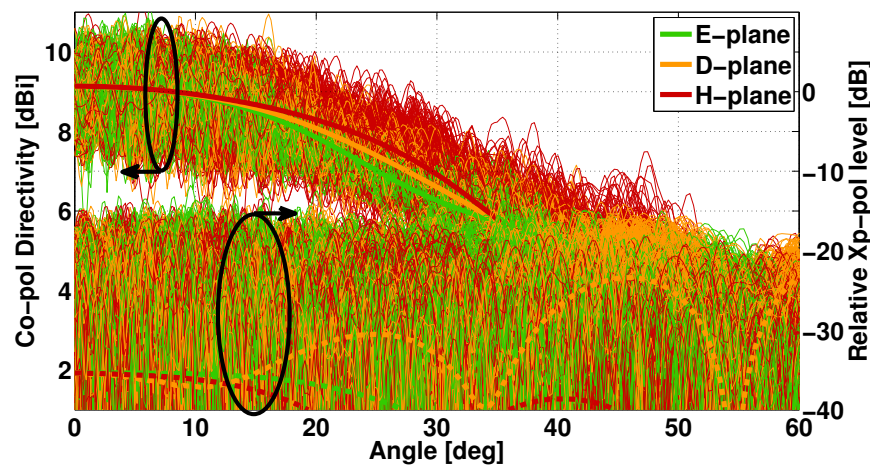

Fig. 6: Simulated embedded element patterns compared to the isolated horn antenna pattern (thick lines).

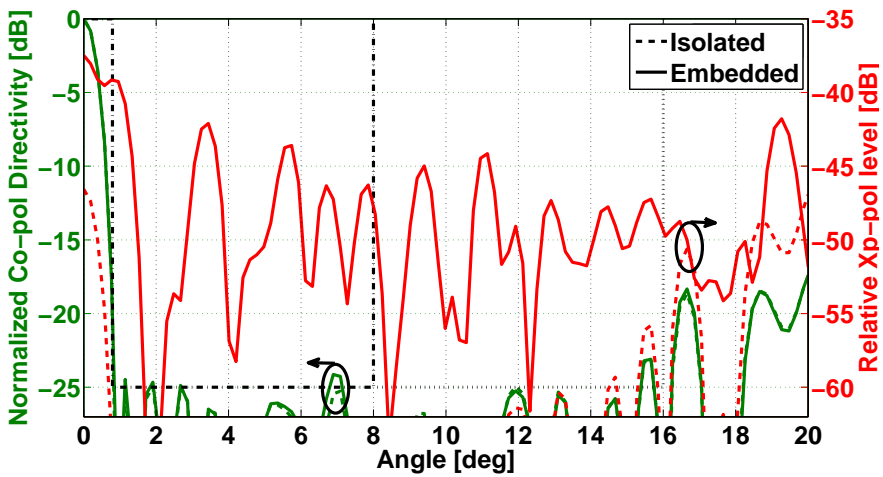

Fig. 7: The co-polarization (green) and cross-polarization (red) components of the far-field patterns (in the D-plane) of the sparse array of 385 pipe horn elements as computed assuming the IEPs (dashed line) and the EEPs obtained from the MoMCBFM simulations (solid line).

[2] R. L. Haupt, "Thinned arrays using genetic algorithms," IEEE Trans. Antennas Propag., vol. 42, no. 7, pp. 993-999, 1994.

[3] M. M. Khodier and C. G. Christodoulou, "Linear array geometry synthesis with minimum sidelobe level and null control using particle swarm optimization," IEEE Trans. Antennas Propag., vol. 53, no. 8, pp. 2674-2679, 2005.

[4] Óscar Quevedo-Teruel and E. Rajo-Iglesias, "Ant colony optimization in thinned array synthesis with minimum sidelobe level," IEEE Antennas Wireless Propag. Lett., vol. 5, no. 1, pp. 349-352, 2006.

[5] G. G. Roy, S. Das, P. Chakraborty, and P. N. Suganthan, "Design of non-uniform circular antenna arrays using a modified invasive weed optimization algorithm," IEEE Trans. Antennas Propag., vol. 59, no. 1, pp. 110-118, 2011.

[6] G. Oliveri, F. Caramanica, and A. Massa, "Hybrid ads-based techniques for radio astronomy array design," IEEE Trans. Antennas Propag., vol. 59, no. 6, pp. 1817-1827, 2009.

[7] Y. Liu, Z. Nie, and Q.-H. Liu, "Reducing the number of elements in a linear antenna array by the matrix pencil method," Antennas and Propagation, IEEE Transactions on, vol. 56, no. 9, pp. 2955-2962, 2008.

[8] G. Oliveri, M. Donelli, and A. Massa, "Linear array thinning exploiting almost difference sets," IEEE Trans. Antennas Propag., vol. 57, no. 12, pp. 3800-3812, 2009.

[9] D. Caratelli and M. Vigano, "A novel deterministic synthesis technique for constrained sparse array design problems," IEEE Trans. Antennas Propag., vol. 59, no. 11, pp. 4085-4093, Nov 2011.

[10] A. Ishimaru, "Unequally spaced arrays based on the poisson sum formula," IEEE Trans. Antennas Propag., vol. 62, no. 4, pp. 1549-1554, April 2014.

[11] W. P. M. N. Keizer, "Large planar array thinning using iterative $\mathrm{fft}$ techniques," IEEE Trans. Antennas Propag., vol. 57, no. 10, pp. 33593362, Oct 2009

[12] O. Bucci, S. Perna, and D. Pinchera, "Advances in the deterministic synthesis of uniform amplitude pencil beam concentric ring arrays," IEEE Trans. Antennas Propag., vol. 60, no. 7, pp. 3504-3509, 2012.

[13] P. Angeletti, G. Toso, and G. Ruggerini, "Array antennas with jointly optimized elements positions and dimensions part ii: Planar circular arrays," IEEE Trans. Antennas Propag., vol. 62, no. 4, pp. 1627-1639, April 2014.

[14] C. Bencivenni, M. Ivashina, R. Maaskant, and J. Wettergren, "Design of maximally sparse antenna arrays in the presence of mutual coupling," IEEE Antennas Wireless Propag. Lett., vol. 159-162, pp. 1-1, 2015.

[15] G. Oliveri, F. Caramanica, and A. Massa, "On the impact of mutual coupling effects on the psll performances of ads thinned arrays," Progress In Electromagnetics Research B, vol. 17, pp. 293-308, 2009.

[16] L. Cen, Z. L. Yu, and W. Ser, "Antenna array synthesis in presence of mutual coupling effect for low cost implementation," in Integrated Circuits, ISIC '09, 2009, pp. 360-363.

[17] A. F. Morabito, A. R. Lagana, and T. Isernia, "Isophoric array antennas with a low number of control points: a 'size tapered' solution,", Progress In Electromagnetics Research Letters, vol. 36, pp. 121-131, 2013.

[18] O. Bucci, T. Isernia, S. Perna, and D. Pinchera, "Isophoric sparse arrays ensuring global coverage in satellite communications," IEEE Trans. Antennas Propag., vol. 62, no. 4, pp. 1607-1618, April 2014.

[19] G. Prisco and M. D’Urso, "Maximally sparse arrays via sequential convex optimizations," IEEE Antennas Wireless Propag. Lett., vol. 11, pp. 192-195, 2012.

[20] S. E. Nai, W. Ser, Z.-L. Yu, and H. Chen, "Beampattern synthesis for linear and planar arrays with antenna selection by convex optimization," IEEE Trans. Antennas Propag., vol. 58, no. 12, pp. 3923-3930, 2010.

[21] P. Ingvarson and J. Zackrisson, "Swedish space antenna projects," in EuCAP 2013, April 2013, pp. 3161-3165.

TABLE III: Element positions defined by polar coordinates $\left(\rho_{n}\right.$ and $\left.\phi_{n}\right)$, excitations $\left(w_{n}\right)$ and their relative change (in superscript) after including MC for the base sector of the layout in Fig. 1. Elements marked with an $\mathrm{x}$ are not present.

\begin{tabular}{|c|c|c|c|c|c|c|c|c|c|c|c|c|c|c|c|c|c|}
\hline & 0.0 & 6.4 & 8.3 & 10.8 & $\mathrm{x}^{10.8}$ & 10.8 & 12.7 & $14.6^{-0.1}$ & 16.6 & 16.6 & $\mathrm{x}^{16.6}$ & 17.2 & 19.1 & 20.4 & 20.4 & 22.9 & 22.9 \\
\hline r & $24.8^{x}$ & 24.8 & 24.8 & 26.1 & 27.4 & 28.6 & 28.6 & 30.6 & $31.8^{x}$ & 31.8 & 31.8 & 31.8 & 31.8 & 32.5 & $32.5^{x}$ & 34.4 & 34.4 \\
\hline$\varepsilon$ & 35.7 & 35.7 & 35.7 & $35.7^{x}$ & 38.8 & 38.8 & 38.8 & 39.5 & 39.5 & 41.4 & 43.9 & 47.1 & 49.7 & 49.7 & $49.7^{-1.3}$ & $49.7^{\mathrm{x}}$ & 49.7 \\
\hline & - & 20.2 & 43.3 & 30.4 & $x^{38.4}$ & 11.9 & 41.6 & $26.4^{-2.0}$ & 13.0 & 32.0 & $\mathrm{x}^{26.8}$ & 44.2 & 23.3 & 7.7 & 37.3 & 18.1 & 26.9 \\
\hline 0 & $0.6^{x}$ & 36.3 & 8.7 & 22.5 & 28.8 & 6.5 & 38.5 & 21.1 & $32.9^{x}$ & 2.3 & 12.1 & 28.4 & 42.8 & 38.4 & $7.5^{x}$ & 19.6 & 24.6 \\
\hline$\theta$ & 14.1 & 30.9 & 43.8 & $2.0^{x}$ & 10.0 & 18.1 & 26.9 & 31.6 & $44.6^{-44.3}$ & 22.5 & $34.9^{+0.7}$ & 43.5 & 13.0 & 17.6 & $43.6^{+0.6}$ & $2.6^{\mathrm{x}}$ & 35.5 \\
\hline & 0.0 & $-3.5^{+0.1}$ & $-4.3^{+1.0}$ & $-5.3^{+1.4}$ & $x^{-7.9}$ & $-4.9^{+1.6}$ & $-3.7^{+0.4}$ & $-7.1^{+1.8}$ & $-9.7^{+2.4}$ & $-4.1^{+1.2}$ & $x^{-6.4}$ & $-3.5^{+0.7}$ & $-6.1^{-0.1}$ & $-6.5^{+1.8}$ & $-4.5^{+1.2}$ & $-5.0^{-0.4}$ & $-5.9^{+1.7}$ \\
\hline & $-12.8^{\mathrm{x}}$ & $-7.2^{+2.3}$ & $-3.3^{+0.4}$ & $-7.7^{+1.7}$ & $-9.4^{+3.0}$ & $-4.7^{+0.8}$ & $-5.0^{-0.3}$ & $-5.8^{+0.3}$ & $-6.0^{x}$ & $-6.0^{+1.5}$ & $-4.5^{-0.4}$ & $-6.9^{+1.3}$ & $-6.7^{+1.1}$ & $-6.0^{-0.3}$ & $-8.6^{x}$ & $-4.6^{+0.2}$ & $-5.2^{+0.9}$ \\
\hline f & $-4.5^{-0.2}$ & $-4.3^{-1.2}$ & $-4.7^{+0.2}$ & $-8.7^{x}$ & $-8.2^{+2.3}$ & $-6.9^{+1.7}$ & $-7.9^{+2.5}$ & $-7.2^{+0.9}$ & $-6.4^{+0.8}$ & $-11.6^{+3.7}$ & $-8.6^{+2.4}$ & $-8.7^{+0.8}$ & $-6.6^{-0.3}$ & $-9.3^{+1.7}$ & $-11.2^{+3.9}$ & $-10.9^{x}$ & $-12.3^{+4.8}$ \\
\hline
\end{tabular}

